\title{
Características físicas do solo e rendimento de grãos de milho em função de culturas
}

\section{de cobertura}

\author{
Soil physical characteristics and corn grain yield as a function of cover crops \\ Características físicas del suelo y rendimiento de grano de maíz en función de los cultivos de \\ cobertura
}

Recebido: 16/12/2021 | Revisado: 22/12/2021 | Aceito: 24/12/2021 | Publicado: 05/01/2022

\author{
Éllen Lemes Silva \\ ORCID: https://orcid.org/0000-0001-5649-5055 \\ Universidade Estadual do Oeste do Paraná, Brasil \\ E-mail: ellen_cbba@hotmail.com \\ Deonir Secco \\ ORCID: https://orcid.org/0000-0002-3042-159X \\ Universidade Estadual do Oeste do Paraná, Brasil \\ E-mail: deonir.secco@ unioeste.br \\ Araceli Ciotti de Marins \\ ORCID: https://orcid.org/0000-0001-8932-7015 \\ Federal University of Technology, Brasil \\ E-mail: araceli@utfpr.edu.br \\ Doglas Bassegio \\ ORCID: https://orcid.org/0000-0001-6628-8594 \\ Universidade Estadual do Oeste do Paraná, Brasil \\ E-mail: doglas.bassegio@unioeste.br \\ Mayra Beatriz Semiano Castro \\ ORCID: https://orcid.org/0000-0002-2818-6623 \\ Universidade Estadual do Oeste do Paraná, Brasil \\ E-mail: mayrabscastro@gmail.com
}

\begin{abstract}
Resumo
O conhecimento das propriedades físicas do solo e o potencial das culturas denominadas "recuperadoras" de estrutura é importante para as tomadas de decisão sobre o manejo agrícola, assim, o estudo envolvendo plantas de cobertura, são importantes quando se objetiva a sustentabilidade dos cultivos. Desta forma, o presente trabalho teve como objetivo avaliar o impacto do cultivo de plantas de cobertura na estrutura de um Latossolo argilosos sob sistema plantio direto e os seus reflexos no rendimento de grãos de milho. Os tratamentos foram constituídos por seis espécies de cobertura de inverno: aveia branca, aveia preta, centeio, aveia preta + nabo forrageiro, aveia preta + tremoço branco, aveia preta + ervilha, além do sistema de plantio direto tradicional (testemunha). O delineamento experimental foi inteiramente casualizado com 4 repetições em parcelas experimentais de $20 \times 16 \mathrm{~m}$. Foram coletadas amostras indeformadas de solo nas camadas de 0,00-0,10 m, 0,1-0,20 m e 0,20-0,30 m, após dessecação das espécies de cobertura e após a colheita do milho, sobre as quais implantou-se a cultura do milho. O rendimento de grãos de milho foi avaliado com 3 repetições, em área de $13,5 \mathrm{~m}^{2}$, e a umidade dos grãos foi corrigida a $13 \%$. As médias dos tratamentos foram comparadas pelo teste Tukey a 5\% de significância, com a utilização do programa Sisvar. As espécies de cobertura que apresentaram maior eficiência na reestruturação do solo foram os consórcios aveia preta + tremoço branco e aveia preta + ervilha forrageira. Quanto ao rendimento de grãos de milho, a aveia branca foi o tratamento que proporcionou o maior rendimento de grãos de milho, não diferindo dos tratamentos centeio, aveia preta + nabo, aveia preta + tremoço branco e do sistema plantio direto tradicional, evidenciando às boas condições de solo e clima da área experimental.
\end{abstract}

Palavras-chave: Espécies de cobertura do solo; Estrutura do solo; Rendimento de grãos do milho.

\begin{abstract}
The knowledge of the physical properties of the soil and the potential of the cultures called "restoratives" of structure is important for decision making about agricultural management, thus, the study involving cover crops are important when aiming at the sustainability of the crops. Thus, this study aimed to evaluate the impact of cover crops on the structure of a clayey Oxisol under no-tillage system and its effects on corn grain yield. The treatments consisted of six winter cover species: white oats, black oats, rye, black oats + radish, black oats + white lupine, black oats + peas, in addition to the traditional no-tillage system (control). The experimental design was completely randomized with 4 replications in experimental plots of 20x16 m. Undisturbed soil samples were collected in layers of 0.00-0.10 m, 0.1-
\end{abstract}


$0.20 \mathrm{~m}$ and $0.20-0.30 \mathrm{~m}$, after desiccation of the cover species and after corn harvesting, on the which the corn crop was implemented. Corn grain yield was evaluated with 3 replications, in an area of $13.5 \mathrm{~m}^{2}$, and grain moisture was corrected to $13 \%$. Treatment means were compared using the Tukey test at 5\% significance, using the Sisvar program. The cover crops that showed greater efficiency in soil restructuring were the intercropping black oat + white lupine and black oat + forage pea. As for corn grain yield, white oat was the treatment that provided the highest corn grain yield, not differing from rye, black oat + turnip, black oat + white lupine and the traditional no-tillage system, showing good results. soil conditions and climate of the experimental area.

Keywords: Land cover species; Soil structure; Corn grain yield.

\section{Resumen}

El conocimiento de las propiedades físicas del suelo y el potencial de los cultivos denominados "reconstituyentes" de estructura es importante para la toma de decisiones sobre manejo agrícola, por lo que el estudio que involucre cultivos de cobertura es importante cuando se busca la sustentabilidad de los cultivos. Por lo tanto, este estudio tuvo como objetivo evaluar el impacto de los cultivos de cobertura en la estructura de un Oxisol arcilloso bajo un sistema de labranza cero y sus efectos en el rendimiento de grano de maíz. Los tratamientos consistieron en seis especies de cobertura invernal: avena blanca, avena negra, centeno, avena negra + rábano, avena negra + altramuz blanco, avena negra + guisantes, además del sistema tradicional de labranza cero (control). El diseño experimental fue completamente al azar con 4 repeticiones en parcelas experimentales de 20x16 m. Se recolectaron muestras de suelo inalterado en capas de 0,00-0,10 m, 0,1-0,20 my 0,20-0,30 m, después de la desecación de las especies de cobertura y después de la cosecha de maíz, sobre las cuales se implementó el cultivo de maíz. Se evaluó el rendimiento de grano de maíz con 3 repeticiones, en un área de 13,5 $\mathrm{m}^{2}$, y se corrigió la humedad del grano al 13\%. Las medias de los tratamientos se compararon mediante la prueba de Tukey al 5\% de significancia, utilizando el programa Sisvar. Los cultivos de cobertura que mostraron mayor eficiencia en la reestructuración del suelo fueron los cultivos intercalados de avena negra + lupino blanco y avena negra + guisante forrajero. En cuanto al rendimiento de grano de maíz, la avena blanca fue el tratamiento que proporcionó mayor rendimiento de grano de maíz, sin diferenciarse del centeno, avena negra + nabo, avena negra + altramuz blanco y el sistema tradicional de labranza cero, mostrando buenos resultados. del área experimental.

Palabras clave: Especies de cobertura terrestre; Estructura del suelo; Rendimiento de grano de maíz.

\section{Introdução}

O intensivo tráfego de máquinas agrícolas sobre o solo pode afetar negativamente a estrutura do solo, favorecendo a ocorrência de erosões e dificultando o crescimento e desenvolvimento das espécies cultivadas, tornando-se assim, de suma importância a avaliação do cultivo de espécies denominadas "recuperadoras" da estrutura do solo (Ruffato et al., 2019 Tokura et al., 2018, Spliethoff et al., 2020; Bareta Junior et al., 2021; Tokura et al., 2021; Castro et al., 2021).

O conhecimento e a compreensão dos efeitos de espécies de cobertura sobre uma possível melhoria do solo nas propriedades físicas, como porosidade e densidade, são de grande importância na busca pela sustentabilidade na agricultura (Pilatti et al., 2018).

Os efeitos benéficos para o solo, quando se utilizam plantas de cobertura, são numerosos, como a ciclagem de nutrientes, fixação de nitrogênio, quebra do ciclo de pragas e doenças, estruturação do solo e adição de matéria orgânica. Isso faz com que a cultura subsequente seja mais capaz de expressar seu potencial produtivo (Silveira et al., 2019).

Estudos com implantação de espécies de cobertura do solo com vigoroso sistema radicular em diferentes sistemas de manejo do solo são de suma importância, devido a diversidade de espécies capazes de produzir diferentes quantidades de resíduos vegetais que após decomposição podem alterar positivamente os atributos físicos e consequentemente aumentar o rendimento dos grãos (Tokura et al., 2016).

O Brasil representa o terceiro maior produtor mundial de milho com uma safra anual 91.08 milhões de toneladas, em que o Paraná é responsável por 14.62 milhões de toneladas, com uma produtividade média de 5944 kg ha-1 (Conab, 2018). Desta forma, o objetivo deste trabalho é o de avaliar a eficiência de espécies de cobertura de inverno na estrutura de um Latossolo argiloso e seus reflexos no rendimento de grãos na cultura do milho (Zea mays). 


\section{Metodologia}

O experimento foi realizado na estação experimental de Santa Tereza do Oeste/PR no Instituto Agronômico do Paraná- IAPAR. Os tratamentos constaram de seis parcelas em sistema plantio direto cultivadas com espécies de cobertura vegetal de inverno de forma isolada ou em consórcio: aveia branca (AB), aveia preta (AP), centeio (CE), aveia preta + nabo forrageiro $(\mathrm{AP}+\mathrm{NF})$, aveia preta + tremoço branco $(\mathrm{AP}+\mathrm{TB})$, aveia preta + ervilha forrageira $(\mathrm{AP}+\mathrm{EF})$, além do sistema plantio direto tradicional (SPDT-Testemunha) em parcelas de $20 \times 16 \mathrm{~m}$.

A área experimental, vinha sendo cultivada com sistema de plantio direto, há pelo menos 18 anos. Antes da implantação do experimento, foi realizada a análise química para caracterização inicial do solo (Tabela 1).

Tabela 1. Caracterização química inicial do solo antes da implantação do experimento na camada de 0-0,20 m no ano de 2010.

\begin{tabular}{|c|c|c|c|c|c|c|c|c|c|c|c|}
\hline $\mathrm{P}$ & $\mathrm{C}$ & $\mathrm{pH}$ & $\mathrm{Al}^{3+}$ & $\mathrm{H}^{+}+\mathrm{Al}^{3+}$ & $\mathrm{Ca}^{2+}$ & $\mathrm{Mg}^{2+}$ & $\mathrm{K}^{+}$ & SB & CTC & $\mathrm{V}$ & $\mathrm{Al}^{*}$ \\
\hline $\mathrm{mg} \mathrm{dm}_{3}^{-}$ & $\mathrm{g} \mathrm{dm}^{-3}$ & $\mathrm{CaCl}_{2}$ & \multicolumn{7}{|c|}{---- } & \multicolumn{2}{|c|}{-----\%----- } \\
\hline 23,6 & 32,72 & 4,70 & 0,32 & 9,00 & 4,20 & 3,00 & 0,44 & 7,64 & 16,64 & 45,91 & 4,02 \\
\hline
\end{tabular}

Fonte: Autores.

O solo da área experimental é classificado como Latossolo Vermelho Distroférrico típico (LVdf) de textura argilosa (Santos, 2018). Os resultados da análise granulométrica das três camadas de solo se encontram na Tabela 2.

Tabela 2. Granulometria do solo da área experimental nas três camadas avaliadas.

\begin{tabular}{|c|c|c|c|}
\hline \multirow{3}{*}{$\begin{array}{c}\text { Camada } \\
\text { (m) }\end{array}$} & \multicolumn{3}{|c|}{ Frações Granulométricas } \\
\hline & Areia & Argila & Silte \\
\hline & \multicolumn{3}{|c|}{ 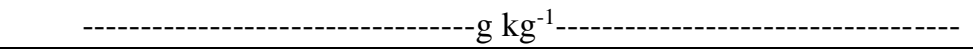 } \\
\hline $0,00-0,10$ & 44,9 & 561,1 & 394,1 \\
\hline $0,10-0,20$ & 38,7 & 641,9 & 319,4 \\
\hline $0,20-0,30$ & 24,7 & 706,2 & 269,1 \\
\hline
\end{tabular}

Fonte: Autores.

Para a determinação da densidade, espaço poroso (porosidade total, macroporosidade e microporosidade) e condutividade hidráulica saturada do solo, foram coletadas amostras indeformadas em três camadas: 0,00-0,10; 0,10-0,20 e 0,20-0,30 m, sempre considerando a profundidade intermediária da camada visada, utilizando cilindros de dimensões 0,05 m de altura e $0,05 \mathrm{~m}$ de diâmetro. Foram coletadas amostras em duplicata em cada camada, a fim de obter amostras representativas, considerando a grande dispersão dos atributos físicos e prevenindo perda de amostras durante a realização dos procedimentos laboratoriais.

Para a avaliação da densidade do solo e macroporosidade, foi utilizada uma coluna de areia que permite a extração seletiva de água a tensões pré-estabelecidas Reinert e Reichert (2006). Posteriormente as amostras foram pesadas e saturadas novamente por 24 horas para a determinação da condutividade hidráulica saturada em permeâmetro de carga constante. Os cálculos para obtenção da condutividade hidráulica do solo foram realizados conforme metodologia preconizada pela Embrapa (1997).

A semeadura do milho foi realizada no dia 08/03/2018 com o híbrido 90XB06 Bt, sendo que as sementes foram tratadas com Vitavax - Thiram 0,3 L $100 \mathrm{~kg}^{-1}$ de sementes. Na semeadura o espaçamento foi de $0,90 \mathrm{~m}$ entre linhas, perfazendo um total de 6 sementes por metro linear. Sem adubação de base, com adubação nitrogenada de $200 \mathrm{~kg}$ ha-1 de uréia e adubação com potássio de $100 \mathrm{~kg}$ de Cloreto de potássio ha ${ }^{-1}$. 
A colheita do milho foi realizada manualmente, onde se avaliou o rendimento de grãos em 3 linhas de 5 metros, com 3 repetições (área de 13,5 $\mathrm{m}^{2}$ ) por parcela. As sementes foram limpas, e o peso foi corrigido para 13\% de umidade.

A análise estatística constou da análise da variância e as médias dos tratamentos foram comparadas pelo teste de Tukey a 5 \% de significância no software Sisvar 5.6.

\section{Resultados e Discussão}

Nas Tabelas 3 e 4 são apresentados os valores médios da densidade do solo (DS), porosidade total (PT), microporosidade (Micro), macroporosidade (Macro) e condutividade hidráulica saturado (Ksat) de amostras de solos nas camadas de 0,00-0,10, 0,10-0,20 e 0,20-0,30 m antes da implantação (2018a) e após a colheita da cultura do milho (2018b).

Tabela 3. Valores médios da densidade do solo (DS) e condutividade hidráulica saturada (Ksat) nas camadas de 0,00-0,10, 0,10-0,20, 0,20-0,30 m, antes da implantação (2018a) e após a colheita da cultura do milho (2018b). AB: aveia branca; AP: aveia preta; $\mathrm{CE}$ : centeio; $\mathrm{AP}+\mathrm{NF}$ : aveia preta + nabo forrageiro; $\mathrm{AP}+\mathrm{TB}$ : aveia preta + tremoço branco; AP+EF: aveia preta + ervilha forrageira; SPDT: sistema de plantio direto tradicional (testemunha). Médias seguidas pelas mesmas letras minúscula na coluna e maiúscula na linha, não diferem entre si pelo Teste de Tukey a 5\% de significância.

\begin{tabular}{|c|c|c|c|c|}
\hline \multirow[t]{3}{*}{ Tratamento } & \multicolumn{2}{|c|}{$\mathrm{DS}\left(\mathrm{Mg} \mathrm{m}^{-3}\right)$} & \multicolumn{2}{|c|}{ Ksat $\left(\mathrm{mm} \mathrm{h}^{-1}\right)$} \\
\hline & \multicolumn{2}{|c|}{$0,00-0,10 \mathrm{~m}$} & \multirow[b]{2}{*}{$2018 \mathrm{a}$} & \multirow[b]{2}{*}{ 2018d } \\
\hline & $2018 \mathrm{a}$ & 2018d & & \\
\hline $\mathrm{AB}$ & $1,06 \mathrm{aA}$ & $1,01 \mathrm{aA}$ & $11,48 \mathrm{bA}$ & $82,29 \mathrm{aA}$ \\
\hline AP & $1,00 \mathrm{aA}$ & $1,00 \mathrm{aA}$ & $65,26 \mathrm{abA}$ & $45,72 \mathrm{aA}$ \\
\hline $\mathrm{CE}$ & $0,99 \mathrm{aA}$ & $0,95 \mathrm{aA}$ & $346,62 \mathrm{aA}$ & $225,94 \mathrm{aA}$ \\
\hline $\mathrm{AP}+\mathrm{NF}$ & $0,98 \mathrm{aA}$ & $0,97 \mathrm{aA}$ & $159,60 \mathrm{abA}$ & $113,10 \mathrm{aA}$ \\
\hline $\mathrm{AP}+\mathrm{TB}$ & $1,10 \mathrm{aA}$ & $1,00 \mathrm{aA}$ & $16,73 \mathrm{bA}$ & $41,56 \mathrm{aA}$ \\
\hline $\mathrm{AP}+\mathrm{EF}$ & $1,05 \mathrm{aA}$ & $0,98 \mathrm{aA}$ & $106,07 \mathrm{abA}$ & $136,19 \mathrm{aA}$ \\
\hline SPDT & $1,05 \mathrm{aA}$ & $1,06 \mathrm{aA}$ & $98,97 \mathrm{abA}$ & $52,78 \mathrm{aA}$ \\
\hline \multicolumn{5}{|c|}{$0,10-0,20 \mathrm{~m}$} \\
\hline $\mathrm{AB}$ & $1,09 \mathrm{aA}$ & $1,06 \mathrm{aA}$ & $31,90 \mathrm{aA}$ & $73,09 \mathrm{abA}$ \\
\hline AP & $1,11 \mathrm{aA}$ & $1,06 \mathrm{aA}$ & $4,77 \mathrm{aA}$ & $100,49 \mathrm{abA}$ \\
\hline $\mathrm{CE}$ & $1,12 \mathrm{aA}$ & $1,09 \mathrm{aA}$ & $69,71 \mathrm{aB}$ & $253,38 \mathrm{aA}$ \\
\hline $\mathrm{AP}+\mathrm{NF}$ & $1,13 \mathrm{aA}$ & $1,05 \mathrm{aA}$ & 75,87aA & $78,45 \mathrm{abA}$ \\
\hline $\mathrm{AP}+\mathrm{TB}$ & $1,16 \mathrm{aA}$ & $1,04 \mathrm{aB}$ & $28,47 \mathrm{aA}$ & $107,70 \mathrm{abA}$ \\
\hline $\mathrm{AP}+\mathrm{EF}$ & $1,18 \mathrm{aA}$ & $0,97 \mathrm{aB}$ & $14,61 \mathrm{aA}$ & $57,33 \mathrm{bA}$ \\
\hline SPDT & $1,16 \mathrm{aA}$ & $1,11 \mathrm{aA}$ & $85,48 \mathrm{aA}$ & $40,31 \mathrm{bA}$ \\
\hline \multicolumn{5}{|c|}{$0,20-0,30 \mathrm{~m}$} \\
\hline $\mathrm{AB}$ & $1,01 \mathrm{bA}$ & $0,90 \mathrm{bB}$ & $15,48 \mathrm{aB}$ & $124,87 \mathrm{aA}$ \\
\hline AP & $1,08 \mathrm{abA}$ & $1,05 \mathrm{aA}$ & $1,17 \mathrm{aA}$ & $62,54 \mathrm{aA}$ \\
\hline $\mathrm{CE}$ & $1,14 \mathrm{abA}$ & $1,06 \mathrm{aA}$ & $19,59 \mathrm{aA}$ & $79,77 \mathrm{aA}$ \\
\hline $\mathrm{AP}+\mathrm{NF}$ & $1,15 \mathrm{aA}$ & $1,04 \mathrm{abB}$ & 7,36aA & $26,67 \mathrm{aA}$ \\
\hline $\mathrm{AP}+\mathrm{TB}$ & $1,13 \mathrm{abA}$ & $0,98 \mathrm{abB}$ & $34,34 \mathrm{aA}$ & $52,86 \mathrm{aA}$ \\
\hline $\mathrm{AP}+\mathrm{EF}$ & $1,18 \mathrm{aA}$ & $1,07 \mathrm{aB}$ & $10,20 \mathrm{aA}$ & $61,84 \mathrm{aA}$ \\
\hline SPDT & $1,15 \mathrm{abA}$ & $1,09 \mathrm{aA}$ & $59,46 \mathrm{aA}$ & $26,87 \mathrm{aA}$ \\
\hline
\end{tabular}

Fonte: Autores.

Pode-se observar na Tabela 3, quanto a DS na camada de 0,00-0,10 m que não houve diferença entre os tratamentos e períodos avaliados, possivelmente devido ao acúmulo de matéria orgânica no solo, assim como bioporos deixados pela senescência do sistema radicular das coberturas vegetais cultivadas anteriormente. Isso pode ocorrer também devido ao 
revolvimento do solo no momento de semeadura, onde ocorre maior atividade biológica e mais ciclos de umedecimento e secagem (Silva et al., 2003). Isso pode ser justificado analisando-se o valor médio inferior da DS nessa camada, se comparada com as demais.

Na profundidade de $0,10-0,20 \mathrm{~m}$ os tratamentos $\mathrm{AP}+\mathrm{TB}$ e $\mathrm{AP}+\mathrm{EF}$, foram os que proporcionaram redução de valores de DS de 1,15 Mg m-3 para 1,04 Mg m-3 e de 1,18 Mg m-3 para 1,07 Mg m-3 respectivamente, pelo fato da aveia ser uma gramínea com o sistema radicular fasciculado, formando um emaranhado de bioporos após a senescência destas raízes nas camadas de subsuperfície do solo. Quanto ao tremoço e ervilhaca por serem leguminosas tem o sistema radicular profundo e vigoroso o que contribui para o aumento dos bioporos e da matéria orgânica, o que reduz a DS e aumentam a sua PT. Ressaltando desta forma a importância do uso de diferentes espécies de cobertura por meio da rotação de culturas para esta camada que é considerada a mais crítica em áreas manejadas sob sistema plantio direto, devido a concentração das deformações impostas pelo tráfego de máquinas e implementos agrícolas.

Entretanto, na camada 0,20-0,30 $\mathrm{m}$ de profundidade os tratamentos que tiveram decréscimo nos valores médios da DS foram $\mathrm{AP}+\mathrm{TB}, \mathrm{AP}+\mathrm{EF}, \mathrm{AB}$ e $\mathrm{AP}+\mathrm{NF}$ com valores indo de 1,01 Mg m-3 para 0,90 Mg m-3, 1,15 Mg m-3 para 1,04 Mg m-3, 1,13 Mg m-3 para 0,98 Mg m-3 e 1,18 Mg m-3 para 1,07 Mg m-3 nesta respectiva ordem, podendo ser justificado quanto ao sistema radicular deixado por essas plantas de cobertura. No que se refere aos períodos avaliados, pode-se ressaltar que antes da implantação da cultura, o tratamento de $\mathrm{AB}(1,01 \mathrm{Mg} \mathrm{m}-3)$ foi o que apresentou menor valor, não diferindo dos tratamentos de AP (1,08 Mg m-3), CE (1,14 Mg m-3), AP+TB (1,13 Mg m-3) e SPDT (1,15 Mg m-3), diferindo-se da AP+NF (1,15 Mg $\mathrm{m}-3)$ e $\mathrm{AP}+\mathrm{EF}$ (1,18 Mg m- 3). No período após o cultivo o menor valor foi encontrado para $\mathrm{AB}(0,90 \mathrm{Mg} \mathrm{m}-3)$, não diferindo da AP+NF (1,04 Mg m-3) e AP+TB (0,98 Mg m-3), diferindo-se da AP (1,05 Mg m-3), CE (1,06 Mg m-3), AP+EF (1,07 Mg m-3) e SPDT (1,09 Mg m- 3).

Nota-se que a DS apresentou a menor média geral (1,01 Mg m-3) na camada de 0,00-0,10 m, já na camada de 0,100,20m apresentou a maior média (1,10 Mg m-3) de DS. Porém, de acordo com Camargo e Alleoni (1997), até mesmo o maior valor da densidade do solo (1,18 Mg m-3) encontrado no presente experimento foi abaixo do considerados crítico para o crescimento radicular, que de acordo com Silva et al., Reichert (2000), em solos argilosos seriam acima de 1,35 Mg m-3.

Estes resultados, diferem dos resultados encontrados por Pilatti et al. (2018), que conclui que as espécies de cobertura não forneceram uma melhoria significativa no estado estrutural do solo. Bem como o apresentado por Da Silveira (2019) que ressalta que as características físicas do solo como DS, PT, Micro e Macro não mostraram diferenças significativas entre os tratamentos envolvendo espécies de cobertura do solo nessa mesma área experimental.

Entretanto Stone e Silveira (2001) encontraram diferenças significativas na DS e PT trabalhando com diferentes sistemas de manejo do solo, durante o período de 3 anos. Spera et al. (2004), observaram diferenças na DS ao conduzirem seus experimentos por 8 anos em sistema de produção de culturas produtoras de grãos e forrageiras sob pastejo, num Latossolo Vermelho distrófico típico, em Passo Fundo (RS).

Uma das formas de reduzir os efeitos negativos da compactação do solo tem sido o uso de espécies de cobertura do solo com sistema radicular mais vigoroso e profundo, que são capazes de reduzir a densidade e aumentar o espaço poroso do solo (RUFFATO et al., 2019).

Quanto ao Ksat para os períodos avaliados pode-se observar na camada de 0,00-0,10 m, antes do cultivo do milho, que o tratamento com maior média foi $\mathrm{CE}\left(346,62 \mathrm{~mm} \mathrm{~h}^{-1}\right)$, que não diferiu da $\mathrm{AP}\left(65,26 \mathrm{~mm} \mathrm{~h}^{-1}\right), \operatorname{AP}+\mathrm{NF}\left(159,60 \mathrm{~mm} \mathrm{~h}^{-1}\right)$, AP+EF $\left(106,07 \mathrm{~mm} \mathrm{~h}^{-1}\right)$ e SPDT $\left(98,97 \mathrm{~mm} \mathrm{~h}^{-1}\right)$ e diferenciou-se de AB $\left(11,48 \mathrm{~mm} \mathrm{~h}^{-1}\right)$ e AP+TB $\left(16,73 \mathrm{~mm} \mathrm{~h}^{-1}\right)$.

Entretanto, na camada de 0,10-0,20m observa-se que o CE foi o que proporcionou aumento da média, de $69,71 \mathrm{~mm} \mathrm{~h}$ 1 para $253,38 \mathrm{~mm} \mathrm{~h}^{-1}$, demonstrando desta forma a diferença entre as plantas de cobertura quanto a interferência por meio do sistema radicular nos parâmetros físicos do solo. Apõs o cultivo do milho o tratamento que apresentou o maior valor também 
foi o de $\mathrm{CE}\left(253,38 \mathrm{~mm} \mathrm{~h}^{-1}\right)$, não diferindo dos tratamentos de $\mathrm{AB}\left(73,09 \mathrm{~mm} \mathrm{~h}^{-1}\right)$, AP $\left(100,49 \mathrm{~mm} \mathrm{~h}^{-1}\right), \mathrm{AP}+\mathrm{NF}\left(78,45 \mathrm{~mm} \mathrm{~h}^{-}\right.$ $\left.{ }^{1}\right)$ e AP+TB $\left(107,70 \mathrm{~mm} \mathrm{~h}^{-1}\right)$, distinguindo-se da AP+EF $\left(57,33 \mathrm{~mm} \mathrm{~h}^{-1}\right)$ e SPDT $\left(40,31 \mathrm{~mm} \mathrm{~h}^{-1}\right)$.

Já na camada de 0,20-0,30 m o tratamento $A B$ foi o que teve acréscimo partindo de $15,48 \mathrm{~mm} \mathrm{~h}^{-1}$ para $124,87 \mathrm{~mm} \mathrm{~h}^{-1}$, o que pode ser justificado pelo grande volume de raízes fasciculadas. Estes resultados corroboram aos encontrados por Tokura et al (2016) que demonstraram a ocorrência das diferenças significativas entre os tratamentos para o Ksat em todas as camadas do solo.

Percebe-se também que ocorreu redução da Ksat à medida que aumentou a profundidade do solo, em que a média geral dos tratamentos para as camadas foram de: $109,05 \mathrm{~mm} \mathrm{~h}^{-1}$ para a camada de $0,00-0,10 \mathrm{~m}, 72,97 \mathrm{~mm} \mathrm{~h}^{-1}$ para $0,10-0,20 \mathrm{~m}$ e de $41,64 \mathrm{~mm} \mathrm{~h}^{-1}$ para $0,20-0,30 \mathrm{~m}$.

Quanto ao coeficiente de variação (CV), o mesmo variou de 109,05\% a 125,39\%, onde até mesmo a menor variação na estrutura do solo, implica diretamente em mudança deste parâmetro, sendo que de acordo com Mesquita e Moraes (2004) relata que, geralmente, os CV's dos métodos de determinação da Ksat são maiores que 70\%.

$\mathrm{Na}$ Tabela 4 pode-se observar quanto a porosidade total (PT) na camada de 0,00-0,10 m que o tratamento de AP+TB foi o que proporcionou aumento de 57,27\% para 61,09\%. Na camada de 0,10-0,20 m as plantas de cobertura AP+TB e AP+EF propiciaram aumento de $55,07 \%$ para $59,78 \%$ e de $54,43 \%$ para $62,50 \%$, respectivamente. Enquanto que, na camada de 0,20 $0,30 \mathrm{~m}$ os tratamentos que propiciaram incrementos na $\mathrm{PT}$ foram $\mathrm{AP}+\mathrm{TB}, \mathrm{AB}, \mathrm{AP}+\mathrm{NF}, \mathrm{AP}+\mathrm{EF}$, com valores, respectivamente de $57,68 \%$ para $63,36 \%, 62,00 \%$ para $66,14 \%, 56,75 \%$ para $61,20 \%$ e $55,60 \%$ para $59,79 \%$.

Quanto aos períodos avaliados observa-se que na camada de 0,20-0,30 m, antes do cultivo do milho, o tratamento de $\mathrm{AB}(62,00 \%)$ foi o que se destacou com a média mais alta, não diferindo da AP (59,54\%), CE (57,29\%), AP+TB (57,68\%) e SPDT (56,93\%) e diferenciando-se da AP+NF (56,75\%) e AP+EF (55,60\%). Após a colheita o tratamento de AB foi o que teve o maior valor de $\mathrm{PT}(66,14 \%)$, não diferindo da $\mathrm{AP}+\mathrm{NF}(61,20 \%)$ e $\mathrm{AP}+\mathrm{TB}(63,36 \%)$, distinguindo-se da $\mathrm{AP}(60,66 \%)$, CE $(60,34 \%), \mathrm{AP}+\mathrm{EF}(59,79 \%)$ e SPDT $(59,10 \%)$.

Uma possível explicação para esses resultados, pode estar associado ao fato das plantas de cobertura combinadas/consorciadas, como no caso da $\mathrm{AP}+\mathrm{NF}, \mathrm{AP}+\mathrm{TB}$ e $\mathrm{AP}+\mathrm{EF}$, terem a junção das gramíneas (sistema radicular fasciculado) com leguminosas (sistema radicular profundo e vigoroso) atuaram de forma mais positiva no incremento da porosidade do solo.

Analisando-se os valores médios gerais da PT, observa-se que são inversamente proporcionais aos apresentados pela DS, de modo que a camada inicial (0,00-0,10 m) apresentou o menor valor de DS e maior valor de PT, enquanto que na camada de 0,10-0,20 m, com maiores valores de Ds e menores de PT (Tabelas 3 e 4), o que já era esperado.

De acordo com Andrade et al., 2009, solos com 50\% de porosidade total são indicativos de boa qualidade física, como os valores encontrados neste trabalho estão todos acima de 50\%, isso indica que os sistemas radiculares dessas espécies de coberturas promoveram benefícios na estrutura do solo para a cultura sucessora. 
Tabela 4. Valores médios da porosidade total (PT), microporosidade (Micro) e macroporosidade (Macro) do solo nas camadas de 0,00-0,10, 0,10-0,20 e 0,20-0,30 m, antes da implantação (2018a) e após a colheita da cultura do milho (2018b). AB: aveia branca; AP: aveia preta; $\mathrm{CE}$ : centeio; $\mathrm{AP}+\mathrm{NF}$ : aveia preta + nabo forrageiro; $\mathrm{AP}+\mathrm{TB}$ : aveia preta + tremoço branco; $\mathrm{AP}+\mathrm{EF}$ : aveia preta + ervilha forrageira; SPDT: sistema de plantio direto tradicional (testemunha). Médias seguidas pelas mesmas letras minúscula na coluna e maiúscula na linha, não diferem entre si pelo Teste de Tukey a 5\% de significância. Médias de tratamentos seguidas de mesmas letras, minúscula na coluna e maiúscula na linha, não diferem entre si pelo Teste de Tukey a $5 \%$ de significância.

\begin{tabular}{|c|c|c|c|c|c|c|}
\hline Tratamento & PT $(\%)$ & & Micro (\%) & \multicolumn{3}{|c|}{ Macro $(\%)$} \\
\hline \multicolumn{7}{|c|}{$0,00-0,10 \mathrm{~m}$} \\
\hline & $2018 \mathrm{a}$ & $2018 d$ & $2018 \mathrm{a}$ & $2018 d$ & $2018 \mathrm{a}$ & $2018 d$ \\
\hline $\mathrm{AB}$ & $58,62 \mathrm{aA}$ & $60,64 \mathrm{aA}$ & $42,32 \mathrm{bA}$ & $44,99 \mathrm{aA}$ & $16,30 \mathrm{aA}$ & $15,65 \mathrm{aA}$ \\
\hline AP & $61,16 \mathrm{aA}$ & $61,16 \mathrm{aA}$ & $48,13 \mathrm{aA}$ & $46,64 \mathrm{aA}$ & $13,03 \mathrm{aA}$ & $14,52 \mathrm{aA}$ \\
\hline $\mathrm{CE}$ & $61,42 \mathrm{aA}$ & $62,98 \mathrm{aA}$ & $43,34 \mathrm{abB}$ & $47,31 \mathrm{aA}$ & $18,08 \mathrm{aA}$ & $15,67 \mathrm{aA}$ \\
\hline $\mathrm{AP}+\mathrm{NF}$ & $61,89 \mathrm{aA}$ & $62,02 \mathrm{aA}$ & $46,05 \mathrm{abA}$ & $46,96 \mathrm{aA}$ & $15,85 \mathrm{aA}$ & $15,06 \mathrm{aA}$ \\
\hline $\mathrm{AP}+\mathrm{TB}$ & $57,27 \mathrm{aB}$ & $61,09 \mathrm{aA}$ & $44,23 \mathrm{abA}$ & $46,79 \mathrm{aA}$ & $13,04 \mathrm{aA}$ & $14,31 \mathrm{aA}$ \\
\hline $\mathrm{AP}+\mathrm{EF}$ & $59,13 \mathrm{aA}$ & $61,75 \mathrm{aA}$ & 46,39abA & $44,44 \mathrm{aA}$ & $12,74 \mathrm{aB}$ & $17,31 \mathrm{aA}$ \\
\hline SPDT & $59,22 \mathrm{aA}$ & $58,75 \mathrm{aA}$ & $44,07 \mathrm{abA}$ & $43,07 \mathrm{aA}$ & $15,15 \mathrm{aA}$ & $15,68 \mathrm{aA}$ \\
\hline \multicolumn{7}{|c|}{$0,10-0,20 \mathrm{~m}$} \\
\hline $\mathrm{AB}$ & $57,92 \mathrm{aA}$ & $59,03 \mathrm{aA}$ & $43,49 \mathrm{aA}$ & $41,84 \mathrm{aA}$ & $14,43 \mathrm{aA}$ & $17,19 \mathrm{aA}$ \\
\hline AP & $57,04 \mathrm{aA}$ & $58,93 \mathrm{aA}$ & $40,84 \mathrm{aA}$ & $41,61 \mathrm{aA}$ & $16,20 \mathrm{aA}$ & $17,32 \mathrm{aA}$ \\
\hline $\mathrm{CE}$ & $56,69 \mathrm{aA}$ & $57,82 \mathrm{aA}$ & $38,04 \mathrm{aA}$ & $39,86 \mathrm{aA}$ & $18,65 \mathrm{aA}$ & $17,96 \mathrm{aA}$ \\
\hline $\mathrm{AP}+\mathrm{NF}$ & $56,55 \mathrm{aA}$ & $59,37 \mathrm{aA}$ & $38,52 \mathrm{aA}$ & $41,56 \mathrm{aA}$ & $18,03 \mathrm{aA}$ & $17,82 \mathrm{aA}$ \\
\hline $\mathrm{AP}+\mathrm{TB}$ & $55,07 \mathrm{aB}$ & $59,78 \mathrm{aA}$ & $41,83 \mathrm{aA}$ & $42,94 \mathrm{aA}$ & $13,24 \mathrm{aB}$ & $16,84 \mathrm{aA}$ \\
\hline $\mathrm{AP}+\mathrm{EF}$ & $54,43 \mathrm{aB}$ & $62,50 \mathrm{aA}$ & $39,94 \mathrm{aB}$ & $47,21 \mathrm{aA}$ & $14,49 \mathrm{aA}$ & $15,29 \mathrm{aA}$ \\
\hline SPDT & $55,29 \mathrm{aA}$ & $57,00 \mathrm{aA}$ & $37,15 \mathrm{aA}$ & $41,08 \mathrm{aA}$ & $18,13 \mathrm{aA}$ & $15,92 \mathrm{aA}$ \\
\hline \multicolumn{7}{|c|}{$0,20-0,30 \mathrm{~m}$} \\
\hline $\mathrm{AB}$ & $62,00 \mathrm{aB}$ & $66,14 \mathrm{aA}$ & $46,06 \mathrm{aA}$ & $50,11 \mathrm{aA}$ & $15,94 \mathrm{aA}$ & $16,03 \mathrm{aA}$ \\
\hline AP & $59,54 \mathrm{abA}$ & $60,66 \mathrm{bA}$ & $44,24 \mathrm{aA}$ & $42,41 \mathrm{bcA}$ & $15,30 \mathrm{aA}$ & $18,25 \mathrm{aA}$ \\
\hline $\mathrm{CE}$ & $57,29 \mathrm{abA}$ & $60,34 \mathrm{bA}$ & $41,15 \mathrm{aA}$ & $43,66 a b c A$ & $16,14 \mathrm{aA}$ & $16,69 \mathrm{aA}$ \\
\hline $\mathrm{AP}+\mathrm{NF}$ & $56,75 \mathrm{bB}$ & $61,20 \mathrm{abA}$ & $40,25 \mathrm{aA}$ & $43,14 a b c A$ & $16,49 \mathrm{aA}$ & $18,06 \mathrm{aA}$ \\
\hline $\mathrm{AP}+\mathrm{TB}$ & $57,68 \mathrm{abB}$ & $63,36 \mathrm{abA}$ & $42,61 \mathrm{aB}$ & 49,16abA & $15,07 \mathrm{aA}$ & $14,20 \mathrm{aA}$ \\
\hline $\mathrm{AP}+\mathrm{EF}$ & $55,60 \mathrm{bB}$ & $59,79 \mathrm{bA}$ & $39,81 \mathrm{aA}$ & $41,55 \mathrm{cA}$ & $15,79 \mathrm{aA}$ & $18,24 \mathrm{aA}$ \\
\hline SPDT & $56,93 \mathrm{abA}$ & $59,10 \mathrm{bA}$ & $39,36 \mathrm{aA}$ & $40,69 \mathrm{cA}$ & $17,57 \mathrm{aA}$ & $18,41 \mathrm{aA}$ \\
\hline
\end{tabular}

Fonte: Autores.

Em relação ao volume de microporos na camada de 0,00-0,10m ressalta-se que o tratamento de CE proporcionou aumento de valor de 43,34\% para 47,31\%, já na camada de 0,10-0,20 m, a AP+EF foi o que gerou acréscimo de 39,94\% para $47,21 \%$ e na camada de $0,20-0,30 \mathrm{~m}$ a AP+TB o acréscimo foi de $42,61 \%$ para $49,16 \%$.

Em relação às épocas avaliadas pode-se observar na camada de 0,00-0,10 $\mathrm{m}$ antes do cultivo do milho, que AP apresentou o maior microporosidade $(48,13 \%)$ não ocorrendo diferença significativa do CE (43,34\%), AP+NF (46,05\%), AP+TB (44,23\%), AP+EF (46,39\%) e SPDT (44,07\%), divergindo do observado na AB (42,32\%). Estes dados corroboram aos resultados encontrados no estudo realizado pela Tokura et al., 2016, com espécies de cobertura, que ressalta que as diferenças significativas entre os tratamentos ocorreram para microporosidade na camada de 0,00-0,10 m.

Na camada de 0,20-0,30m pode ressaltar que a $\mathrm{AB}(50,11 \%)$ foi o que apresentou a maior média de microporosidade, não diferindo do CE (43,66\%), AP+NF (43,14\%) e AP+TB (49,16\%) e distinguiu-se da AP (42,41\%), AP+EF (41,55\%) e SPDT $(40,69 \%)$.

Quanto aos valores de macroporosidade na camada de 0,00-0,10 m, observa-se que o tratamento de AP+EF proporcionou aumento de $12,74 \%$ para $17,31 \%$, já na camada de $0,10-0,20 \mathrm{~m}$, enquanto o tratamento de AP+TB foi de $13,24 \%$ para $16,84 \%$. Todos estes valores médios de macroporosidade estão acima dos valores considerados críticos, pois segundo 
Prevedello (1996) volume de macroporosidade igual ou inferior a 10\% prejudica o desenvolvimento dos cultivos agrícolas. Isso evidencia o bom estado estrutural da área experimental.

Tabela 5. Rendimentos dos grãos de milho em função dos tratamentos envolvendo espécies de cobertura de verão (valores médios de três repetições). Médias de tratamentos seguidas de mesmas letras na coluna, não diferem entre si pelo Teste de Tukey a 5\% de significância. AB: aveia branca; AP: aveia preta; CE: centeio; AP+NF: aveia preta + nabo forrageiro; AP+TB: aveia preta + tremoço branco; AP+EF: aveia preta + ervilha forrageira; SPDT: sistema de plantio direto tradicional (testemunha). Médias seguidas pelas mesmas letras minúscula na coluna e maiúscula na linha, não diferem entre si pelo Teste de Tukey a $5 \%$ de significância.

\begin{tabular}{lc}
\hline Tratamento & Rendimento dos grãos $\left(\mathrm{kg} \mathrm{ha}^{-1}\right)$ \\
\hline $\mathrm{AB}$ & $6952 \mathrm{a}$ \\
$\mathrm{AP}$ & $5620 \mathrm{~b}$ \\
$\mathrm{CE}$ & $6485 \mathrm{ab}$ \\
$\mathrm{AP}+\mathrm{NF}$ & $6421 \mathrm{ab}$ \\
$\mathrm{AP}+\mathrm{TB}$ & $6392 \mathrm{ab}$ \\
$\mathrm{AP}+\mathrm{EF}$ & $5447 \mathrm{~b}$ \\
SPDT & $6487 \mathrm{ab}$ \\
\hline
\end{tabular}

Fonte: Autores.

Na Tabela 5, encontram-se os valores médios do rendimento de grãos de milho nos diferentes tratamentos avaliados. Pode-se observar que o tratamento de $\mathrm{AB}\left(6951 \mathrm{~kg} \mathrm{ha}^{-1}\right)$ foi o que proporcionou o maior valor de rendimento de grãos, não diferindo do CE (6485 $\left.\mathrm{kg} \mathrm{ha}^{-1}\right), \mathrm{AP}+\mathrm{NF}\left(6420 \mathrm{~kg} \mathrm{ha}^{-1}\right)$, AP+TB $\left(6392 \mathrm{~kg} \mathrm{ha}^{-1}\right)$ e SPDT (6487 kg ha-1), diferenciando-se da AP (5619 $\mathrm{kg} \mathrm{ha}^{-1}$ ) e AP+EF (5446 kg ha-1). Estes valores divergem do encontrado por Martins, Gonçalves, Junior (2016) que conclui que o emprego das espécies de cobertura não afetou o rendimento produtivo da cultura do milho.

\section{Conclusão}

As espécies de cobertura de inverno que apresentaram maior eficiência na reestruturação do solo foram os consórcios aveia preta + tremoço branco e aveia preta + ervilha forrageira.

A aveia branca foi o tratamento que propiciou o maior rendimento de grãos de milho, não diferindo dos tratamentos de centeio, aveia preta + nabo forrageiro, aveia preta + tremoço branco e do SPDT, evidenciando às boas condições de solo e clima da área experimental.

\section{Referências}

Andrade, R. D. S., \& Stone, L. F. (2009). Índice S como indicador da qualidade física de solos do cerrado brasileiro. Revista Brasileira de Engenharia Agrícola e Ambiental, 13(4), 382-388.

Bareta Junior, E, da Silva, A. A. P., Sens, T. M. Z. G., Colecha, K., Rampim, L., \& Pott, C. A. (2021). Soil physical properties in variable levels of soil compaction. Research, Society and Development, 10(2), e2341028686-e2341028686

Camargo, O.A., Alleoni, L.R.F. (1997). Compactação do solo e o desenvolvimento de plantas. Potafós. 132p.

Castro, M. B. S., Secco, D., de Marins, A. C., Bassegio, D., de Souza, S. N. M., \& Junior, L. A. Z. (2021). Propriedades físicas do solo e produtividade de grãos de milho cultivo após espécies de cobertura. Research, Society and Development, 10(16), e220101623786-e220101623786.

CONAB - Companhia Nacional de Abastecimento. Acompanhamento da safra Brasileira, v.5-safra 2017/2018-n.6. Sexto levantamento.

Santos, H. G., Jacomine, P. K. T., Dos Anjos, L. H. C., De Oliveira, V. A., Lumbreras, J. F., Coelho, M. R., Cunha, T. J. F. (2018). Sistema brasileiro de classificação de solos. Brasília, DF: Embrapa, 
Research, Society and Development, v. 11, n. 1, e21511124823, 2022

(CC BY 4.0) | ISSN 2525-3409 | DOI: http://dx.doi.org/10.33448/rsd-v11i1.24823

Empresa Brasileira de Pesquisa Agropecuária, Embrapa (1997). Centro Nacional de Pesquisa de Solos. Manual de métodos de análise de solo.

Martins, D., Gonçalves, C. G., \& Silva, A. C. D. (2016). Coberturas mortas de inverno e controle químico sobre plantas daninhas na cultura do milho. Revista Ciência Agronômica, 47, 649-657.

Mesquita, M. D. G. B. D. F., \& Moraes, S. O. (2004). A dependência entre a condutividade hidráulica saturada e atributos físicos do solo. Ciência Rural, 34(3), 963-969.

Pilatti, M. A., Secco, D., Zanão Jr, L. A., Marins, A. D., Tokura, L. K., \& Villa, B. D. (2018). Structural alterations of Paraná's oxisols by cover crops. Journal of Agricultural Science, 10(9) 180-190.

Prevedello, C. L. Física do solo com problemas resolvidos. Curitiba, Saeafs, 1996, 446p.

Reinert, D. J., \& Reichert, J. M. (2006). Coluna de areia para medir a retenção de água no solo: protótipos e teste. Ciência Rural, 36, 1931-1935.

Ruffato, G. G., Secco, D., Zanão Junior, L. A., Tokura, L. K., de Marins, A. C., Villa, B. D., \& do Nascimento, L. F. J. (2019). Structuring of a haplortox by cover crops and their effects on the yield of soybean grains. Journal of Agricultural Science, 11(5), 309-313.

Silva, R. B. D., Junior, D., Silva, F. A. D. M., \& Fole, S. M. (2003). O tráfego de máquinas agrícolas e as propriedades físicas, hídricas e mecânicas de um Latossolo dos Cerrados. Revista Brasileira de Ciência do Solo, 27, 973-983.

Silva, V. D., Reinert, D. J., \& Reichert, J. M. (2000). Densidade do solo, atributos químicos e sistema radicular do milho afetados pelo pastejo e manejo do solo. Revista Brasileira de Ciência do Solo, 24, 191-199.

Silveira, L. D., Secco, D., Zanão Júnior, L. A., Tokura, L. K., Santos, R. F., Villa, B. D., ... \& Montiel, C. B. (2019). Nitrogen fertilization on a soybean crop grown on black oat and forage pea straw in a clayey Latosol in western Paraná. Journal of Agricultural Science (Toronto), 11(7), 196-204.

Spera, S. T., Santos, H. P., Fontaneli, R. S., \& Tomm, G. O. (2004). Efeitos de sistemas de produção de grãos envolvendo pastagens sob plantio direto nos atributos físicos de solo e na produtividade. Revista Brasileira de Ciência do Solo, 28, 533-542.

Spliethoff, J., Pott, C. A., Rampim, L., Watzlawick, L. F., \& Jadoski, S. O. (2020). Limites de compactação do solo para Ilex paraguariensis. Research, Society and Development, 9(5), e23953101-e23953101.

Stone, L. F., \& Silveira, P. D. (2001). Efeitos do sistema de preparo e da rotação de culturas na porosidade e densidade do solo. Revista Brasileira de Ciência do Solo, 25(2), 395-401.

Tokura, L. K., Secco, D., Júnior, L. A. Z., Siqueira, J. A. C., Alovisi, A. M. T., Barison, A., \& Zin, Z. (2021). Use of cover crops in Oxisol and its effects on yield and soybean oil content. Research, Society and Development, 10(12), e353101220514-e353101220514.

Tokura, L. K., Secco, D., Júnior, L. A. Z., Siqueira, J. A. C., Santos, R. F., Martins, M. F. L., \& Schmatz, M. J. (2017). Structuring potential of some cover crops and crambe in Haplortox under no-tillage system. African Journal of Agricultural Research, 12(24), 2078-2086. 